\title{
Los negocios inmobiliarios en la ciudad de Quito y Las actividades emprendidas por la inmobiliaria Torre Marshall
}

\author{
Fecha de recepción: 27/11/2019 • Fecha de aceptación: 15/01/2020 • Fecha de publicación:10/02/2020
}

\author{
Stephania Freire \\ DK Management Services S.A. \\ stephaniefreirec@gmail.com \\ https://orcid.org/0000-0001-7870-895 \\ Ramiro Freire \\ GMS / Grupo Microsistemas \\ david.freirecif@gmail.com \\ https://orcid.org/0000-0002-3624-2891
}

\section{RESUMEN}

En el mercado habitacional en el Ecuador específicamente en la ciudad de Quito, tanto la oferta, como la demanda reflejan condiciones alentadoras en cuanto a la construcción y comercialización de viviendas. En el presente artículo se detalla un plan de negocios para la Inmobiliaria Torre Marshall, orientado a la comercialización de viviendas y locales comerciales dirigido a clientes de clase media-alta en los sectores más estratégicos y exclusivos de la ciudad de Quito. El estudio se enmarca en un tipo documental, de alcance descriptivo, con un enfoque cuantitativo, puesto que se consideran documentos de diversa índole para describir el mercado de bienes raíces que da soporte al plan de negocio de la inmobiliaria. La principal conclusión es que a nivel nacional es justamente Quito el de mayor proporción de ofertas inmobiliarias, aunque circunstancialmente la demanda se haya frenado debido a la situación económica y política, la recuperación es inminente y expedita. Así mismo, la densidad poblacional de la ciudad es baja, lo cual evidencia su potencial de crecimiento. De allí que el proyecto de la inmobiliaria Torre Marshall acorde a los estudios legales, técnicos y financieros, se 
vislumbre como un excelente negocio, que comenzará con la Torre Marshall como un proyecto piloto, que demuestra la preminencia de construcciones de vivienda vertical para el aprovechamiento del espacio físico urbano, dada la imposibilidad de la cuidad de crecer horizontalmente.

PALABRAS CLAVE: mercado habitacional, plan de negocios, inmobiliaria.

\begin{abstract}
In the housing market in Ecuador specifically in the city of Quito, both the supply and the specific demand encouraged conditions regarding the construction and modifications of homes. This article details a business plan for the Marshall Tower Real Estate, aimed at classifying homes and commercial premises aimed at upper-middle class clients in the most strategic and exclusive sectors of the city of Quito. The study is framed in a documentary type, of descriptive scope, with a quantitative approach, since divergence documents are considered to describe the real estate market that supports the real estate business plan. The main conclusion is that at the national level, Quito is precisely the largest proportion of real estate offers, although circumstantially the demand has slowed due to the economic and political situation, the recovery is imminent and expeditious. Likewise, the population density of the city is low, which shows its growth potential. Hence, the Marshall Tower real estate project agreed to legal, technical and financial studies, is seen as an excellent business, which will begin with the Marshall Tower as a pilot project, which demonstrates the predominance of vertical housing constructions for the use of the urban physical space, given the impossibility of the caregiver to grow horizontally.
\end{abstract}

KEYWORDS: housing market, business plan, real estate 


\section{INTRODUCCIÓN}

La construcción es parte fundamental del desarrollo de un país, representa la imagen de evolución o retroceso económico de una sociedad. El impulso que este sector genera en la economía vincula a varios sectores productivos tales como industrial, energético, forestal, transporte, comunicaciones, comercial, turístico, financiero, etc. que marcan el progreso de una economía. Si un país avanza, el sector de la construcción se desarrollará en esa magnitud, de allí que la importancia de la gestión del agente inmobiliario y su coordinación con las diferentes actividades involucradas para la creación de un inmueble bien raíz, a fin de satisfacer una necesidad habitacional o comercial.

El sector de la construcción se ha caracterizado por ser uno de los mecanismos reactivadores de la economía y por su aporte a la generación de empleo. Es por ello que en los planes del gobierno se le ha concedido especial interés, con objetivos precisos, desde la creación de las unidades de poder adquisitivo constante hasta la canalización específica de los recursos a la construcción de vivienda (Banco de la República, 1992, citado por Restrepo, 2016).

El desarrollo en el Ecuador al igual que en muchos países de Latinoamérica está directamente ligado con el rumbo político que en algunas ocasiones limita o impulsa el crecimiento económico. Desde la inversión del sector público en viviendas populares hasta en las grandes obras de ingeniería reflejan un avance social, generando bienestar en su colectividad. Por otro lado, la inversión privada aporta de manera prominente a la inversión de nuevos proyectos de construcción, sujeta a las oportunidades que ofrezca el medio, relacionadas directamente al crecimiento del PIB, créditos, tasas, oportunidades de inversión, demanda de vivienda, remesas de los inmigrantes, entre otras, factores que están vinculados directamente a la actividad inmobiliaria.

En la actualidad se presenta un incremento migratorio agigantado aparte de los convencionalmente dados por Perú y Colombia se da este fenómeno por parte de Venezuela por la situación que se encuentra atravesando este país. Las grandes ciudades como Quito, Guayaquil, Ambato, entre otras son las que mayor incidencia han tenido en este sentido; por lo que, la construcción de vivienda debe presentar alternativas con perspectivas de beneficio, tomando esto en consideración. La construcción de vivienda vertical permite edificar en menores espacios mayores cantidades de vivienda, esto tiende a contribuir de alguna forma la solución de demanda y ofrecer un aprovechamiento máximo del área de suelos.

La ciudad de Quito presenta complicaciones para crecer horizontalmente debido a sus características orográficas y topográficas propias del lugar; ya que, de expenderse hacia el norte o al sur dichas extensiones estarían ya fuera del cantón Quito, situación que justifica plenamente la ejecución de proyectos de vivienda vertical en sectores específicos apropiados para aprovechar el escaso suelo en condiciones de ser utilizado con menor inversión.

\section{Contextualización espacio temporal del problema}

Los diversos cambios que Ecuador ha experimentado en su economía ha dado paso a que se 
realicen diversidad de ajustes estructurales que de una u otra forma han afectado el crecimiento de varios sectores económicos, dentro de los cuales se considera como uno de los primarios al sector de la construcción por ocupar el tercer lugar en el aporte que representa el PIB, además de que el sector aporta a la reducción de la tasa de desempleo; puesto que, esta actividad es generadora de propulsar varios sectores productivos, afectando el incremento del gasto público incapaz de mantener la liquidez del sistema, provocando que los niveles de crecimiento se conviertan en consumos de los hogares y la formación bruta de capital fijo.

Para definir de una manera más clara la situación del Ecuador en el tema urbano se hace necesario puntualizar, que este país se caracteriza por tener primacía urbana compartida en dos grandes ciudades, en la que se reconocen la persistencia de dos sistemas geográficos con dinámica propia y gran capacidad de concentración de excedentes generados en comparación con otras ciudades y provincias del país, en la que se identifica a Quito por ser capital y estar concentrado en ella la gobernabilidad del país con sus diferentes carteras; así como, el comercio y crecimiento poblacional; mientras que; Guayaquil capital financiera del país por contar con el puerto más importante de la economía, generando una relación entre el sector público y el sector privado que es el fenómeno que determina la concentración de dicho dinamismo en las redes urbanas, junto al manejo de la información de los movimientos de capitales, las ciudades que más experimentan un desarrollo real son las que poseen una fuerte actividad ligada a la iniciativa de sus propios actores. Estas condiciones se cumplen a menor escala en las ciudades de Cuenca, Machala, Ambato, Santo domingo de los T-Sachilas y en la provincia de Manabí, Portoviejo y Manta.

De esto se desprende que estas ciudades de manera proporcional de acuerdo a su importancia económica, socio-cultural y político hayan sido objeto de inmigraciones internas de los diferentes sectores del país y de migraciones externas, provocando un aumento poblacional dimensional específicamente en Quito y Guayaquil, situación que genera un desabastecimiento de vivienda acorde a este crecimiento.

Los estudios de gentrificación, considerada esta como la transformación de un área de clase trabajadora de la ciudad con perspectivas de enclave a una zona de clase media, ya sea esta para un uso residencial o comercial, se han concentrado en procesos de restructuración social y desplazamientos en los centros y áreas de influencia de las ciudades, en el caso de Quito, estos desplazamientos se han concentrado en las zonas periurbanas en las que se ha producido una mayor transformación socio espacial producto de la demanda desmedida del aumento poblacional, lo que obliga al gobierno nacional a tomar las medidas pertinentes. Esto ha obligado a realizar estudios que permitan indagar en el contexto de este proceso expansivo de la ciudad, los posibles efectos de la acción inmobiliaria y municipal en las transformaciones socio espaciales de las zonas ocupadas por los habitantes tradicionales y sectores periféricos y rurales de la ciudad. (Duran, Martí y Mérida, 2016)

Las cifras revelan, por ejemplo, que en los años 80 Quito tenía una superficie de 16196 hectáreas y para el 2016 se triplicó. Sin embargo, la densidad en la mancha urbana durante estos últimos 30 años se ha mantenido: 55 personas por hectárea, lo que evidencia la dispersión de la población en el territorio. La densidad de Quito es una de las más bajas de la región. Bogotá, por ejemplo, tiene 
245 habitantes por hectárea, Buenos Aires cuenta con 142, Lima con 120 y São Paulo con 79. (Cinco desafíos marcan el crecimiento de Quito, 2018)

\section{El mercado inmobiliario}

Según el Fondo Monetario Internacional (2014), el mercado inmobiliario ayuda al desarrollo de un país porque: - Promueve inversiones - Desarrolla bienes inmuebles tangibles - Promueve el bienestar de la sociedad - Contribuye al PIB por medio de bienes, capital, producción y consumo - Genera empleo - Los bienes inmuebles generan valor a través del tiempo - Brinda programas de renovación urbanística, entre otros. Por otro lado, dentro de este mercado se constituye el mercado de viviendas, sector activo y de relevancia dentro de una economía, ya que se considera a la vivienda como un bien de consumo fundamental, siendo un activo importante en la cartera de un gran porcentaje de las familias, además este forma parte de un parte fundamental de la economía del país, manteniendo un vínculo directo con la producción, construcción y mantenimiento de viviendas (Vera y Titelman, 2013).

\section{MÉTODOS Y MATERIALES}

El presente plan de negocios, se enmarca en una investigación documental, de alcance descriptivo, bajo un enfoque cuantitativo

Investigación documental: Se conceptualiza como: Estrategia metodológica de obtención de información, que supone por parte del investigador el instruirse acerca de la realidad objeto de estudio a través de documentos de diferente materialidad (escritos, visuales, numéricos, etc.), con el fin de acreditar las justificaciones e interpretaciones que realiza en el análisis y reconstrucción de un fenómeno que tiene características de historicidad. (Yuni y Urbano, 2014). Este trabajo obtiene la información de documentos, como periódicos, libros, etc.

Estudio descriptivo: buscan especificar las propiedades, características y los perfiles de personas, grupos, comunidades o cualquier otro fenómeno que sea sometido a análisis. Es decir, únicamente pretenden medir o recoger información de manera independiente o conjunta sobre las variables a las que se refieren. (Hernández, Fernández y Baptista y otros, 2010). En el estudio de describe la situación del mercado habitacional en Quito y el plan de negocios d la inmobiliaria Torre Marshall

Enfoque cuantitativo: En este sentido, el método cuantitativo de acuerdo con Hernández, Fernández y Baptista (2010) manifiestan que usan la recolección de datos para probar hipótesis, con base en la medición numérica y el análisis estadístico, para establecer patrones de comportamiento y probar teorías, puesto que se analizan mediciones numéricas que proporcionan información específica de la realidad a través de la cual se puede explicar y predecir el proceso.

Método deductivo: Permite llegar a conclusiones específicas tomando como punto de partida la percepción de la mayoría de nuestros potenciales clientes y de esta manera ofrecer soluciones tendientes a satisfacer necesidades acordes a sus planteamientos tanto en el aspecto comercial como en el de vivienda. 


\section{RESULTADOS}

A nivel nacional respecto a la oferta inmobiliaria, se observa que es justamente en la ciudad de Quito, en donde existe la mayor oferta de proyectos residenciales, superando con creces las demás ciudades importantes de

\section{Movimiento en oferta de proyectos residenciales}

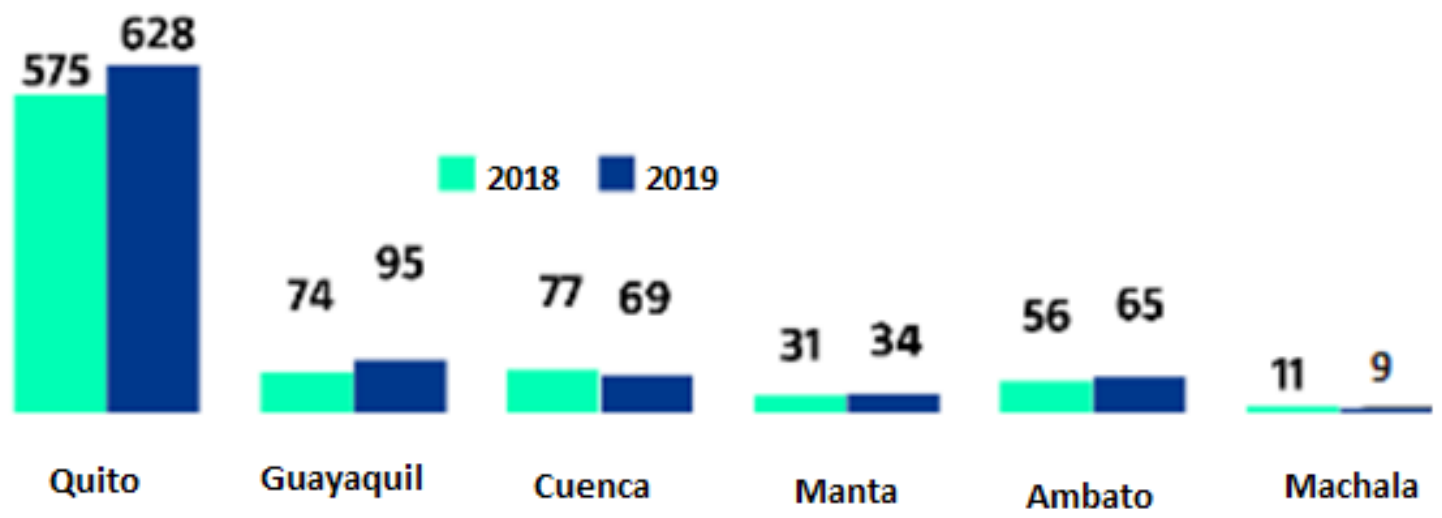

Figura 1. Oferta inmobiliaria. Fuente: Market Watch (Oferta de vivienda crece, pero la demanda baja. 2019)

Sin embargo, aunque la oferta ha aumentado, la demanda se ha frenado por la situación económica y la incertidumbre sobre el futuro político del país, según explica Germán Carvajal, de la División Inmobiliaria de Market Watch. (Oferta de vivienda crece, pero la demanda baja. 2019)

Según datos exhibidos en la feria de vivienda "mi casa clave" reflejan con claridad lo que acontece en la actividad inmobiliaria en el Distrito metropolitano de Quito. Según los datos exhibidos esta feria recibió 27622 visitantes quienes pudieron analizar ofertas de 542 proyectos inmobiliarios presentados por 110 empresas superior a la feria realizada en el año 2017. Así mismo de estos proyectos enunciados se refleja los valores del total de negociaciones cerradas en esta feria que alcanzó una cifra \$13627.995. En el cuadro x también se observa que es Quito Norte el sector que tiene la mayor proporción de reservas, lo cual reitera que la ciudad de Quito presenta un buen movimiento inmobiliario.

Cuadro 1

Reservas para proyectos inmobiliarios, Año 2018

\begin{tabular}{lll}
\hline SECTOR & RESERVAS & $\%$ \\
\hline QUITO NORTE & $\$ \quad 5.500 .665,20$ & 40 \\
\hline
\end{tabular}




\begin{tabular}{lrrl}
\hline LOS CHILLOS & $\$$ & $3.490 .700,00$ & 26 \\
\hline CALDERON - CARAPUNGO & $\$$ & $1.691 .710,00$ & 12 \\
\hline POMASQUI- MITAD DEL MUNDO & $\$$ & $820.200,00$ & 6 \\
\hline CUMBAYA - TUMBACO & $\$$ & $721.270,00$ & 5 \\
\hline QUITO SUR & $\$$ & $578.600,00$ & 4 \\
\hline QUITO CENTRO - NORTE & $\$$ & $500.100,00$ & 4 \\
\hline TONSUPA & $\$$ & $185.000,00$ & 1 \\
\hline PUEMBO & $\$$ & $105.000,00$ & 1 \\
\hline MANABI & $\$$ & $34.750,00$ & 0 \\
\hline TOTAL & $\mathbf{1 3 6 2 7 9 9 5 , 2 0}$ & $\mathbf{1 0 0}$ \\
\hline
\end{tabular}

Reservas en Feria abril 2018 - Properati. Elaboración propia

\section{Estudio técnico}

\section{Procesos de Organización y Gestión Empresarial}

La creación de la inmobiliaria Marshall está orientada a la comercialización de bienes raíces, siendo el objetivo principal del presente plan de negocios realizar las actividades de venta de locales comerciales y suites del edificio que lleva su propio nombre "Torre Marshal" ubicado en el sector de La Mariscal para progresivamente ampliarse en proyectos definidos de las mismas características.

La localización es en la provincia de Pichincha, ciudad de Quito, parroquia La Mariscal en las calles Gerónimo Carrión 584 y Juan León Mera sector turístico de la ciudad

\section{Organigrama Estructural}

El presente esquema permite determinar funciones, responsabilidades y departamentos con su respectivo nivel jerárquico así como la asignación de funciones a cada uno de ellas a través del cual se alcanzaran los objetivos trazados por la organización en el presente plan de negocios 




Figura 2: Estructura Organigrama funcional. Elaboración propia

\section{Procesos de Servucción}

La actividad del agente inmobiliario está orientada a prestar servicios de mediación, asesoramiento y gestión en la compra, venta, alquiler o sesiones de vivienda y otros bienes inmuebles, tanto para compradores, vendedores, inquilinos o caseros. En esta actividad se debe tomar en consideración la importancia que tiene cada uno de sus clientes a fin de prestarle el asesoramiento debido en base a su experiencia para satisfacer adecuadamente la exigencia de los mismos. 


\section{Cuadro 2}

Descripción del servicio

\begin{tabular}{|c|c|c|c|}
\hline SERVICIO & DETALLE & RESPONSABLE & TIEMPO \\
\hline $\begin{array}{l}\text { Plena } \\
\text { disposición al } \\
\text { cliente }\end{array}$ & $\begin{array}{l}\text { Disponibilidad } \\
\text { integral para } \\
\text { coordinar consultas, } \\
\text { citas, visitas, } \\
\text { atención telefónica, } \\
\text { preparar ofertas, } \\
\text { documentación, } \\
\text { negociar entre las } \\
\text { partes (bajo un trato } \\
\text { cordial) }\end{array}$ & Agente Vendedor & 24 horas \\
\hline $\begin{array}{l}\text { Buscar } \\
\text { viviendas } \\
\text { adecuadas al } \\
\text { comprador }\end{array}$ & $\begin{array}{l}\text { Compatibles con } \\
\text { las necesidades } \\
\text { y presupuesto del } \\
\text { comprador (revisar } \\
\text { constantemente listado } \\
\text { de propiedades) }\end{array}$ & Agente Vendedor & 8 horas \\
\hline $\begin{array}{l}\text { Analizar } \\
\text { mercado } \\
\text { inmobiliario }\end{array}$ & $\begin{array}{l}\text { Definir el verdadero } \\
\text { valor del bien, analizar } \\
\text { si la posible compra } \\
\text { representa buena } \\
\text { opción, intermediario } \\
\text { de ofertas y contra } \\
\text { ofertas }\end{array}$ & $\begin{array}{l}\text { Asistente comercial } \\
\text { y marketing }\end{array}$ & 2 horas \\
\hline $\begin{array}{l}\text { Inspección de } \\
\text { viviendas } \\
\end{array}$ & $\begin{array}{l}\text { Aspecto legal, revisión } \\
\text { y selección }\end{array}$ & $\begin{array}{l}\text { Asesor técnico y } \\
\text { Departamento Legal }\end{array}$ & 1 hora \\
\hline $\begin{array}{l}\text { Publicidad de } \\
\text { viviendas }\end{array}$ & $\begin{array}{l}\text { Promover el mercado } \\
\text { a través de canales } \\
\text { de distribución, } \\
\text { anuncios en guías de } \\
\text { servicio, prensa, radio, } \\
\text { expositores locales, } \\
\text { exhibiciones y ferias, } \\
\text { portales de internet, } \\
\text { redes sociales }\end{array}$ & $\begin{array}{l}\text { Asistente comercial } \\
\text { y marketing }\end{array}$ & 1 hora \\
\hline $\begin{array}{l}\text { Ofertar } \\
\text { financiación } \\
\text { adecuada }\end{array}$ & $\begin{array}{l}\text { Asesorar la mejor } \\
\text { condición de } \\
\text { financiación, costes de } \\
\text { escrituras. }\end{array}$ & $\begin{array}{l}\text { Gerente Comercial } \\
\text { y marketing }\end{array}$ & 1 hora \\
\hline
\end{tabular}




\section{Estrategias del Servicio Inmobiliario.}

El principal valor de servicio y producto es el liderazgo en costos, representa una ventaja potencial competitiva, consiste en ofrecer el mismo producto o servicio a menor costo de la competencia en el mercado, para lograr este objetivo se debe analizar la rentabilidad de todos los actores del mercado, al presentar precios más bajos su resultado tiende a comercializar más producto en menor tiempo, en un proyecto de vivienda en un inicio se deben ofertar porcentajes de descuento considerables al de la competencia. En el proyecto Torre Marshall, las proyecciones de comercialización están orientadas a repotenciar esta estrategia, ya que en el sector los rubros de estos valores son muy elevados, situación que permite aplicar con eficiencia la estrategia.

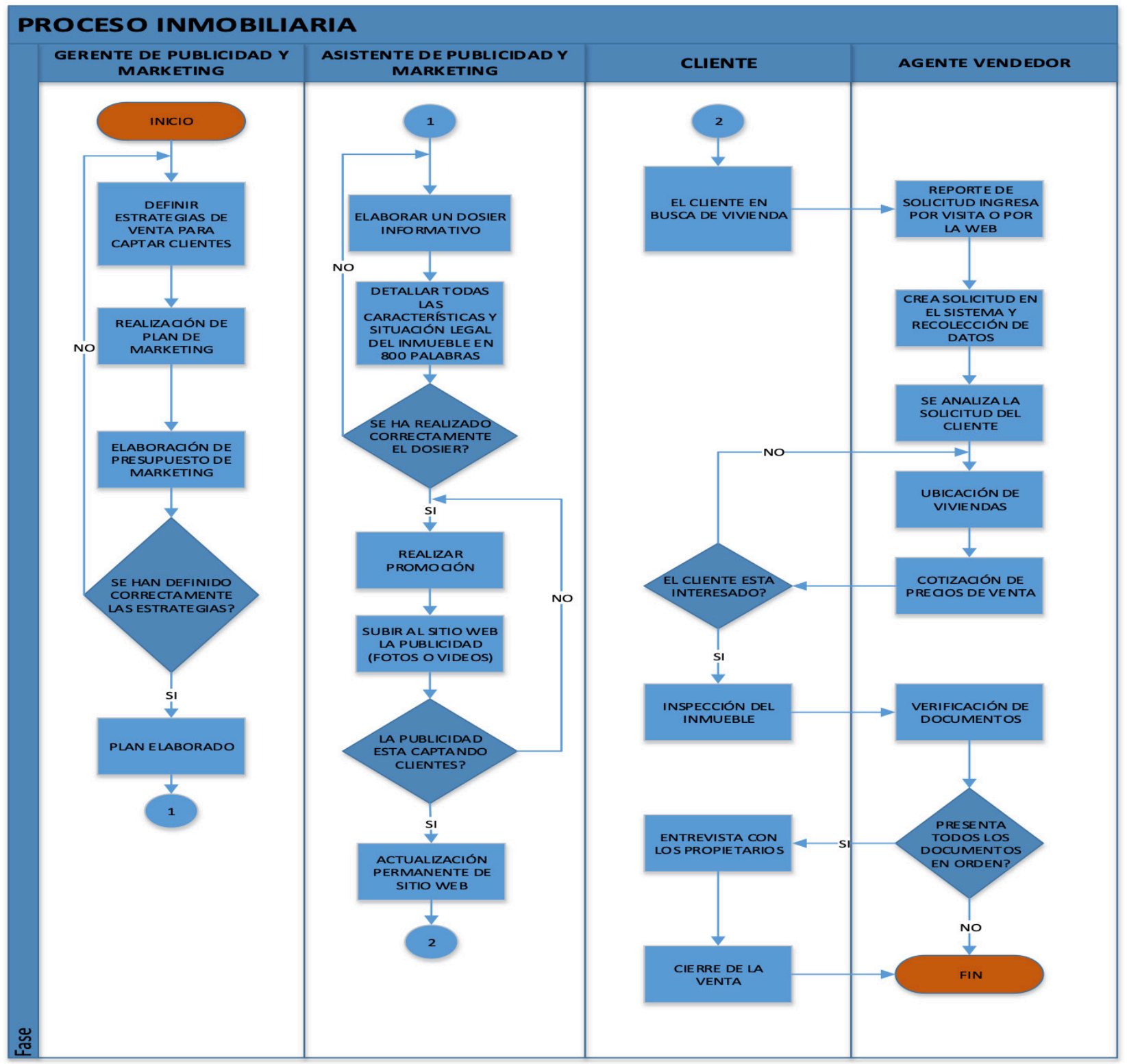

Figura 3. Flujograma del proceso inmobiliario. Elaboración propia 


\section{Aspectos legales}

El cumplimiento de los aspectos legales del negocio inmobiliario se sintetiza a continuación

- Elaborar los estatutos.

- Abrir una cuenta de integración de capital.

- Acudir a Notaria para registrar la escritura pública.

- Llevar la escritura pública a la Superintendencia de Compañías, para la aprobación del estatuto

- Publicar en el diario la resolución entregada por La Superintendencia de Compañías.

- Obtener los permisos municipales la patente municipal y el certificado de cumplimiento de obligaciones

- Inscribir la compañía en el Registro Mercantil

- Realizar el registro del RUC en el Servicio de Rentas Internas (SRI)

\section{Estudio Financiero}

\section{Inversión total del proyecto}

La inversión para el inicio de operaciones de la Inmobiliaria Marshal es de 16097.50 (Dieciséis mil noventa y siete $50 / 100$ ), en los que el $42.39 \%$ serán activos fijos, el $7.89 \%$ activos diferidos y el $49.72 \%$ capital de trabajo.

\section{Cuadro 3}

Inversión total del proyecto

\begin{tabular}{lrr}
\hline INVERSIÓN & DÓLARES & PORCENTAJE \\
\hline ACTIVOS FIJOS & & \\
Propiedades y plantas & 1000,00 & $6,21 \%$ \\
Muebles y ensteres & 3770,00 & $23,42 \%$ \\
Equipos de computación & 2030,00 & $12,61 \%$ \\
Equipos electrónicos & 24,00 & $0,15 \%$ \\
Subtotal & $\mathbf{6 8 2 4 , 0 0}$ & $\mathbf{4 2 , 3 9 \%}$ \\
ACTIVOS DIFERIDOS & & \\
Gastos de constitución & 820,00 & $5,09 \%$ \\
Permisos de funcionamiento & 300,00 & $1,86 \%$ \\
Gastos de puesta en marcha & 150,00 & $0,93 \%$ \\
Subtotal & $\mathbf{1 2 7 0 , 0 0}$ & $\mathbf{7 , 8 9} \%$ \\
CAPITAL DE TRABAJO & & \\
Efectivo & 8003,50 & $49,72 \%$ \\
Subtotal & $\mathbf{8 0 0 3 , 5 0}$ & $\mathbf{4 9 , 7 2 \%}$ \\
TOTAL INVERSIONES & $\mathbf{1 6 0 9 7 , 5 0}$ & $\mathbf{1 0 0 , 0 0 \%}$ \\
\hline
\end{tabular}




\section{Financiamiento del proyecto}

La inversión inicial del proyecto se realizara el 100\% con capital propio.

Cuadro 4

Financiamiento del proyecto

\begin{tabular}{ccc}
\hline FUENTE & VALOR & $\%$ \\
\hline Recursos propios & 16097,50 & $100 \%$ \\
TOTAL & 16097,50 & $100 \%$ \\
\hline
\end{tabular}

Cuadro 5

Presupuesto de costos y gastos

\begin{tabular}{lrrrrr}
\hline & & & & & AÑOS \\
\hline COSTOS Y GASTOS & $\mathbf{1}$ & $\mathbf{2}$ & $\mathbf{3}$ & $\mathbf{4}$ & $\mathbf{5}$ \\
GASTOS DE & & & & & \\
ADMINISTRACIÓN & & & & & \\
Sueldos administrativos & 88560,00 & 88976,23 & 89394,42 & 89814,57 & 90236,70 \\
Amortizaciones & 635,00 & 635,00 & & & \\
Depreciaciones & 887,80 & 887,80 & 887,80 & 887,80 & 887,80 \\
Energía eléctrica & 420,00 & 421,97 & 423,96 & 425,95 & 427,95 \\
Internet & 960,00 & 964,51 & 969,05 & 973,60 & 978,18 \\
Teléfono & 2772,00 & 2785,03 & 2798,12 & 2811,27 & 2824,48 \\
Suministros de oficina & 330,00 & 331,55 & 333,11 & 334,67 & 336,25 \\
Subtotal & $\mathbf{9 4 5 6 4 , 8 0}$ & $\mathbf{9 5 0 0 2 , 1 0}$ & $\mathbf{9 5 4 4 8 , 6 1}$ & $\mathbf{9 5 8 9 7 , 2 2}$ & $\mathbf{9 6 3 4 7 , 9 3}$ \\
Gastos de ventas & & & & & \\
Publicidad & 3000,00 & 3014,10 & 3028,27 & 3042,50 & 3056,80 \\
SUBTOTAL & $\mathbf{3 0 0 0 , 0 0}$ & $\mathbf{3 0 1 4 , 1 0}$ & $\mathbf{3 0 2 8 , 2 7}$ & $\mathbf{3 0 4 2 , 5 0}$ & $\mathbf{3 0 5 6 , 8 0}$ \\
TOTAL COSTOS Y & 97564,80 & 98016,20 & 98476,87 & 98939,71 & 99404,73 \\
GASTOS & & & & & \\
\hline
\end{tabular}


Cuadro 6

Estado de flujo de efectivo

\begin{tabular}{|c|c|c|c|c|c|}
\hline INGRESOS OPERACIONALES & 1 & 2 & 3 & 4 & 5 \\
\hline Recuperación por ventas & 100080,00 & 108656,86 & 128078,67 & 139055,01 & 150972,03 \\
\hline $\begin{array}{l}\text { Parcial } \\
\text { EGRESOS OPERACIONALES }\end{array}$ & 100080,00 & 108656,86 & 128078,67 & 139055,01 & 150972,03 \\
\hline Gastos de Administración & $-94564,80$ & $-95002,10$ & $-95448,61$ & $-95897,22$ & $-96347,93$ \\
\hline Gastos de Ventas & $-3000,00$ & $-3014,10$ & $-3028,27$ & $-3042,50$ & $-3056,80$ \\
\hline & 887,80 & 887,80 & 887,80 & 887,80 & 887,80 \\
\hline An & 635,00 & 635,00 & & & \\
\hline Par & $-96042,00$ & $-96493,40$ & $-97589,07$ & $-98051,91$ & $-98516,93$ \\
\hline $\begin{array}{l}\text { FLUJO OPERACIONAL } \\
\text { EGRESOS NO OPERACIONALES }\end{array}$ & 4038,00 & 12163,46 & 60 & 10 & 5,10 \\
\hline Pas & $-377,28$ & $-1596,10$ & $-4536,59$ & $-6114,70$ & $-7833,58$ \\
\hline $\begin{array}{l}\text { Pago de impuesto a la renta } \\
\text { ACTIVOS FIJOS OPERATIVOS } \\
\text { Propiedades y plantas } \\
\text { Muebles y enseres } \\
\text { Equipos de computación } \\
\text { Equipos electrónicos } \\
\text { Activos diferidos } \\
\text { Capital de trabajo }\end{array}$ & $-470,34$ & $-1989,80$ & $-5655,62$ & $-7622,99$ & $-9765,86$ \\
\hline $\begin{array}{l}\text { Parcial } \\
\text { Valor residual de la inversión fija } \\
\text { Recuperación }\end{array}$ & $-847,62$ & $-3585,90$ & $-10192,21$ & $-13737,69$ & $\begin{array}{r}-17599,44 \\
2385,00 \\
8003,50\end{array}$ \\
\hline JO NO OPERACIONAL & $-847,62$ & $-3585,90$ & $-10192,21$ & $-13737,69$ & $-7210,94$ \\
\hline GENE & 3190,38 & 8577,56 & 20297,38 & 27265,41 & 45244,15 \\
\hline
\end{tabular}

\section{Evaluación financiera}

$$
V A N=\sum_{(1+\text { Tmar })}^{\text {Flujos }} n
$$

\section{Cuadro 7}

\section{Evaluación financiera VAN}

\begin{tabular}{cc}
\hline AÑO & VALOR \\
\hline $\mathbf{0}$ & $-16097,50$ \\
$\mathbf{1}$ & 3190,38 \\
$\mathbf{2}$ & 8577,56 \\
$\mathbf{3}$ & 20297,38 \\
$\mathbf{4}$ & 27265,41 \\
$\mathbf{5}$ & 45244,15 \\
$\mathbf{n}$ & 5 AÑOS \\
$\mathbf{~}$ & 11,63 \\
\hline
\end{tabular}

$\operatorname{VAN}=51894,86$

TIR

$$
T I R=T m+D t\left(\frac{V A N_{\text {MENOR }}}{V A N_{\text {MENOR }}-V A N_{\text {MAYOR }}}\right)
$$


Cuadro 8

Evaluación financieraTIR

\begin{tabular}{cc}
\hline ANOO & VALOR \\
\hline $\mathbf{0}$ & $-16097,50$ \\
$\mathbf{1}$ & 3190,38 \\
$\mathbf{2}$ & 8577,56 \\
$\mathbf{3}$ & 20297,38
\end{tabular}

$T I R=68 \%$

Una vez obtenido los resultados de los cálculos correspondientes al TIR y al VAN, se demuestra que el proyecto es viable o satisfactorio financieramente con una Tasa Interna de Retorno del $68 \%$ $\geq 11.63 \%$ de la TMAR.

\section{Proyecto Torre Marshall}

La imposibilidad de crecer horizontalmente en la ciudad hace aún más difícil la ejecución de proyectos que permitan de forma apropiada solucionar esta necesidad.

Esta la razón que para que en sectores tradicionales de la ciudad de Quito que guardan espacios con viviendas tradicionales con superficies de terreno apropiadas para aplicar construcciones de vivienda vertical en las que dichos espacios pueden ser utilizados para el aprovechamiento de mejores condiciones que abarquen mayor volumen de vivienda en el mismo espacio físico. Este tipo de construcción o edificios ayudan a densificar más la ciudad en menores superficies a lo contrario de un desarrollo horizontal, se reducen los costos de urbanización, existe seguridad ya estatuida, vías de acceso, de líneas de transporte también ya establecidas, entre otros factores, dicho de otra forma este concepto prevé facilidades en todo sentido para la ampliación de vivienda mejorando significativamente el nivel, comodidad y calidad de las condiciones que puedan ofrecerse. Este concepto de construcción vertical forma en la actualidad el total de un proyecto con previsión más factible, económica, estructural en un entorno de ubicación adecuada a la necesidad de demanda en perspectivas de mejores condiciones.

El proyecto innovador de la inmobiliaria Marshal aplicada en el proyecto de suites y locales en un sector privilegiado de la ciudad permitirá demostrar que se pueden utilizar espacios físicos de viviendas antiguas en construcciones verticales para resolver de alguna manera la demanda de vivienda en mejores condiciones que en las periferias de la ciudad, dando un perfil innovador al resto de sectores similares en la ciudad.

Cabe señalar que de acuerdo a las disposiciones legales municipales en el tiempo en que se constituyeron este tipo de vivienda la mayor parte de los espacios físicos que estas abarcan es de $700 \mathrm{~m} 2$ a 900m2 en su mayoría, lo que hace propicio que se emprendan edificaciones de hasta 8 pisos de acuerdo a la ordenanza municipal actual, lo que beneficiara tanto a sus propietarios como 
a la factibilidad de vivienda y locales comerciales en el sector. Por otro lado la inmobiliaria Torre Marshal realizara una investigación exhaustiva referente al tema a fin de aplicar estrategias que conlleven a propiciar la utilización de estos bienes con la perspectiva señalada, pues los resultados serán la muestra clara para que exista una visión de tendencia a este tipo de construcción a dueños y propietarios del sector, coadyuvando de esta manera la necesidad inminente y falencia de estos servicios tan importantes en la ciudad.

De igual manera se pretende aplicar la misma perspectiva de proyecto en otros sectores de la ciudad de Quito y el país que guardan similares condiciones que el proyecto actual.

El proyecto Torre Marshal está localizado en la provincia de Pichincha, ciudad de Quito, parroquia La Mariscal en las calles Gerónimo Carrión 584 y Juan León Mera sector turístico de la ciudad con una superficie total de $712 \mathrm{~m} 2$ y proyección de $4936 \mathrm{~m} 2$ de construcción con tres subsuelos, dos plantas de locales, seis pisos de suites con espacios verdes y áreas comunales, sector en el que se presenta una inminente demandas de vivienda y de locales comerciales. Actualmente en la ciudad como se indicó anteriormente, las viviendas se expanden a las afueras dejando la zona central como un sector de comercio, situación que sucede en este sector, en la que se han implantado en construcciones antiguas acondicionamientos para el efecto; adicionalmente a esto, en el sector no existe una cultura de inversión en el mercado inmobiliario por lo que el presente proyecto plantea movilizar este mercado y aprovechar las condiciones actuales que presenta la economía del país.

De acuerdo a los antecedentes identificados anteriormente se desprende que el presente plan de negocios está considerado en la ciudad con mayor afluencia demográfica poblacional y habitacional del país acompañado de la gran complejidad de restricción de extensión que por su configuración geográfica esta mantiene. La actividad inmobiliaria avizora enfocar su gestión en el centro norte de la ciudad en el que se desarrollan diferentes actividades socio-políticas, culturales, comercio y turísticas de gran importancia.

En este sentido, la creación del plan de negocios para la inmobiliaria Torre Marshall, pretende fomentar a los propietarios de bienes raíces del sector tomen las iniciativas a fin de emprender los mismos tipos de construcción en sus propiedades,

El proyecto de la inmobiliaria edificio Torre Marshal está orientado a la comercialización de locales comerciales y suites a fin de captar personas con recursos económicos medio altos por su ubicación exclusiva, permitiendo disfrutar del confort ajustadas a la nueva tecnología que busca facilitar la vida alrededor de las tareas del hogar, lo que hace que estas sean menos desgastantes, el diseño minimalista y funcional permite tener un ambiente practico integrado y acogedor, con ventajas de seguridad, privacidad e intimidad con vista privilegiada, siendo la mejor opción para parejas de recién casados o con poco número de integrantes, personas solteras, adultos mayores, empresarios, ejecutivos de negocio estudiantes y extranjeros. Las áreas sociales y comunitarias permiten disfrutar de un entorno de servicios y confort a sus actividades personales. La categoría de los locales comerciales darán realce al comercio del entorno. 
Cuadro 9

Descripción de Torre MArshall

\begin{tabular}{lc}
\hline \multicolumn{1}{c}{ DESCRIPCIÓN } & CANTIDAD \\
\hline Subsuelos & 3 \\
\hline Parqueaderos & 63 \\
\hline Bodegas & 27 \\
\hline Locales en Planta baja y Primera planta & 8 \\
\hline Suites en Segunda planta alta & 4 \\
\hline Suites en Tercera planta alta & 4 \\
\hline Dúplex en Tercera planta alta & 2 \\
\hline Suites en Cuarta planta alta & 4 \\
\hline Suites en Quinta planta alta & 3 \\
\hline Suites en Sexta planta alta & 3 \\
\hline Dúplex en Sexta planta alta & 3 \\
\hline Suites en Séptima planta alta & 4 \\
\hline Área comunal en Octava planta alta & 1 \\
\hline
\end{tabular}

\section{Características del Proyecto Torre Marshal}

- Elegante lobby de acceso

- Dos ascensores de última tecnología

- Tres subsuelos de estacionamientos

- Guardianía 24 horas

- Generador eléctrico para áreas comunales, cisterna y suites

- Sistema de seguridad de circuito cerrado de tv y control de accesos

- Fibra óptica con telefonía I.P.

- Paredes de división de suites con mampostería de bloque relleno para evitar ruido.

- Amplios ventanales maximizan la iluminación, ventilación natural y proyección visual de sus alrededores.

- Estructura antisísmica 


\section{DISCUSIÓN}

De la oferta inmobiliaria a nivel nacional, es la ciudad de Quito la que tiene mayor proporción de proyectos residenciales, así mismo, la cuidad ofrece tanto para nacionales, como para extranjeros, condiciones de vida que son atractivas. Pese a que la demanda pueda haber decaído por la situación economía y política del país, se espera una pronta recuperación. De allí que el plan de negocios para la inmobiliaria Torre Marshall tenga una buena proyección en el mercado inmobiliario de la ciudad. Al realizar el estudio técnico, legal y financiero, se observa que dicho plan presenta viabilidad, puesto que se presentan todos los requisitos, la localización, la estructura organizacional, la estimación de flujos de caja, etc. Asimismo los indicadores calculados para realizar la evaluación financiera como el Valor Actual Neto y la Tasa Interna de Retorno resultaron satisfactorios (VAN de aproximadamente 51.000 y una TIR de $69 \%$ )

\section{CONCLUSIONES}

En La ciudad de Quito deben prevalecer las construcciones de vivienda vertical para el aprovechamiento del espacio físico urbano, dada la imposibilidad de la cuidad de crecer horizontalmente. La densidad de Quito es una de las más bajas de la región, es de 55 personas por hectárea, cifra que ha sido constante en los últimos 30 años, lo que evidencia la dispersión de la población en el territorio y su potencial de crecimiento inmobiliario. De allí que el plan de negocios de la inmobiliaria Marshall sea un buen prospecto, además de que se sustenta en una estructura de diseño integrado, cuyos estudios técnico, legal y financiero arrojan resultandos positivos, dado que los indicadores utilizados para la evaluación de proyectos de inversión, como el VAN y la TIR resultan satisfactorios.

De cumplirse de manera eficaz con los objetivos propuestos en el presente plan, aunado a la debida aplicación de estrategias, la empresa alcanzará con seguridad el crecimiento empresarial en el mercado inmobiliario. 


\section{REFERENCIAS BIBLIOGRÁFICAS}

Cinco desafíos marcan el crecimiento de la Quito (6 de dciembre de 2018) El Comercio. (s. f.). Recuperado de https://www.elcomercio.com/actualidad/quito-poblacion-construcciones-crecimiento-barrios.html

Durán, G., Martí, M., \& Mérida, J. (2016). Crecimiento, segregación y mecanismos de desplazamiento en el periurbano de Quito. Íconos - Revista de Ciencias Sociales. https://doi.org/10.17141/iconos.56.2016.2150

Hernández, Fernández y Baptista )2010 Metodóloga de la investigación

Merizalde, R. 2017 Análisis de la evolución del sector de la construcción en el Ecuador en el período 2010 - 2016 y sus efectos en la economía nacional Universidad De Especialidades Espíritu Santo Facultad De Economía Y Ciencias Empresariales Título.. Recuperado de http://repositorio.uees.edu.ec/bitstream/123456789/1763/1/Análisis sector construcción - Final \%284\%29.pdf

Proyecciones Poblacionales IINEC. (s. f.). Recuperado de http://www.ecuadorencifras.gob.ec/proyecciones-poblacionales/

Oferta de vivienda crece, pero la demanda baja, 24 de agosto de 2019 Redacción Economía Diario El Telégrafo Recuperado de https://www.eltelegrafo.com.ec/noticias/economia/4/oferta-de-vivienda-crece-pero-la-demanda-baja

Restrepo D 24 de agosto de 2016 - La importancia del sector de la construcción en materia económica.Hábitat y Desarrollo Urbano La network https://la.network/la-importancia-del-sector-de-la-construccion-en-materia-economica/ 